\title{
TRANSMISSION OF OBLIQUELY INCIDENT SURFACE WAVES THROUGH A NARROW GAP
}

\section{B.N. MANDAL and P.K. KUNDU}

\author{
Department of Applied Mathematics \\ University of Calcutta \\ 92 A.P.C. Road \\ Calcutta - 700009 \\ (Received January 22, 1987)
}

\begin{abstract}
This note is concerned with the transmission of a train of surface water waves obliquely incident on a thin plane vertical barrier with a narrow gap. Within the framework of the linearized theory of water waves, the problem is reduced to the solution of an integral equation which is solved approximately. The transmission and reflection co-efficients are also obtained approximately and represented graphically against the different angles of incidence for fixed wave numbers.
\end{abstract}

KEY WORDS AND PHRASES. Water waves, linearized theory, narrow gap, oblique incidence, reflection and transmission coefficients.

1980 AMS SUBJECT CLASSIFICATION CODE. 76B.

1. INTRODUCTION.

When a train of surface water waves is incident on a plane vertical barrier with a gap, it is partially reflected and partially transmitted. For the case of normal incidence Tuck [1] used the method of matched asymptotic expansion to solve the problem for a gap which is assumed to be narrow in the sense that the depth of submergence of the midsection of the gap is large compared to its breadth. Packham and Williams [2] also considered the same problem and used an integral equation (IE) formulation wherein IE is on the horizontal component of velocity across the gap. The IE is then solved analytically by an approximate method. Porter [3] used a reduction procedure and also an IE formulation (different from the approach of Packham and Williams [2]). Both the methods considered by Porter [3] lead to finding the solution of the same Riemann-Hilbert problem. In the IE formulation considered by Porter [3], the IE is on the difference of potential across the barrier above and below the gap. While Tuck [1] and Porter [3] considered the problem only for deep water, Packham and Williams [2] extended it to water of finite constant depth.

The transmission and reflection coefficients obtained by Porter [3] are in principle exact, but that obtained by Tuck [1] or Packham and Williams [2] are approximate under the assumption that the depth of submergence of the midsection of the gap is assumed to be large compared to its breadth.

In the present note we generalize the problem in deep water to the case of a 
wave train impinging the barrier obliquely. An IE formulation is used to reduce the problem to the solution of an IE involving the unknown horizontal component of velocity across the gap. This IE is reduced to the solution of a system of IE's by an approximate procedure which is successfully used in some related obliquely incident water wave scattering problems involving obstacles in the form of plane vertical barriers (cf. Mandal and Goswami [4], [5], [6]) and a half immersed horizontal circular cylinder (cf. Mandal and Goswami [7]).

2. STATEMENT AND FORMULATION OF THE PROBLEM.

We consider the motion in an inviscid and incompressible fluid occupying the region $y \geq 0$, the mean free surface (FS) coinciding with the plane $y=0,|x|>0$. A plane vertical barrier of infinite horizontal extent with a gap is present in the fluid, the barrier being represented by $x=0$, the gap being situated in the region $a<y<b$. Assuming the motion to be irrotational and simple harmonic in time with cylinder frequency $\sigma$ and of small amplitude, a velocity potential exists which may be described by $\operatorname{Re}\{x(x, y, z)$ exp $(-1 \sigma t)\}$. Under the linearized theory $x$ satisfies the Laplace's equation in the fluid region, the FS condition

$$
\frac{\partial \chi}{\partial y}+K x=0 \text { on } y=0
$$

the barrier condition

$$
\frac{\partial x}{\partial x}=0 \text { on } \mathrm{x}=0,0<\mathrm{y}<\mathrm{a} \text { and } \mathrm{b}<\mathrm{y}<\infty \text {, }
$$

where $K=\frac{\sigma^{2}}{g}, g$ being the gravity.

A train of surface water waves represented by $x_{0}=\exp \{-K y+i K \cos \alpha x+i K \sin \alpha z\}$ is assumed to be incident at an angle $\alpha$ with the normal to the barrier. In view of the geometry of the barrier we can assume,

$$
x(x, y, z)=\Phi(x, y) \exp (i \vee z)
$$

where $v=K \sin \alpha$. Then $\Phi(\mathrm{x}, \mathrm{y})$ satisfies

$$
\begin{aligned}
& \left(\frac{\partial^{2}}{\partial x^{2}}+\frac{\partial^{2}}{\partial y^{2}}-v^{2}\right) \Phi=0 \text { in the fluid region, } \\
& \frac{\partial \Phi}{\partial y}+K \Phi=0 \text { on } y=0, \\
& \frac{\partial \Phi}{\partial x}=0 \text { on } x=0,0<y<a \text { and } b<y<\infty,
\end{aligned}
$$

where $\Phi(x, y)$ and its partial derivatives are continuous everywhere except possible across $\mathrm{x}=0,0<\mathrm{y}<\mathrm{a}$ and $\mathrm{b}<\mathrm{y}<\infty$. We also require that $\Phi$ and $\nabla \Phi$ are bounded everywhere away from the two sharp edges of the wall at the gap, and near these edges $|\nabla \Phi|=O\left(r^{\frac{1}{2}}\right)$ where $r$ denotes the distance from the edges.

Let us write

$$
\Phi(\mathrm{x}, \mathrm{y})=\phi_{\mathrm{o}}(\mathrm{x}, \mathrm{y})+\phi(\mathrm{x}, \mathrm{y})
$$

where $\phi_{0}(x, y)=\exp (-k y+i K \cos \alpha x)$, then $\phi(x, y)$ satisfies $(2.1),(2.2)$ and the edge 
condition stated above. As the incident wave trainexperiences reflection by the barrier and transmission through the gap, the asymptotic behavior of $\Phi(x, y)$ as $|x| \rightarrow \infty$ is given by

$$
\begin{aligned}
& \Phi(\mathrm{x}, \mathrm{y}) \sim \mathrm{T} \phi_{\mathrm{o}}(\mathrm{x}, \mathrm{y}) \text { as } \mathrm{x} \rightarrow \infty \\
& \Phi(\mathrm{x}, \mathrm{y}) \sim \phi_{\mathrm{O}}(\mathrm{x}, \mathrm{y})+\mathrm{R} \phi_{\mathrm{o}}(-\mathrm{x}, \mathrm{y}) \text { as } \mathrm{x} \rightarrow-\infty
\end{aligned}
$$

where $T$ and $R$ are the (complex) transmission and reflection co-efficients respectively.

By an appropriate use of Green's integral theorem in the region $y>0, x>0$ and $y>0, x<0$ we obtain following Mandal and Goswami [6]

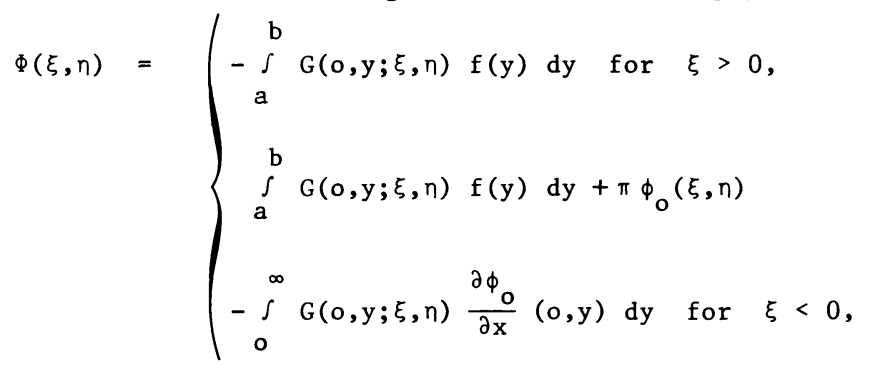

where $f(y)=\frac{\partial \Phi}{\partial x}(o, y), a<y<b$,

and $G(x, y ; \xi, \eta)$ is the Green's function given by Levine [8] as

$$
\begin{aligned}
G(x, y ; \xi, n) & =K_{0}(\nu \rho)-K_{0}\left(\nu \rho^{*}\right) \\
& +2 \int_{\nu}^{\infty} \frac{\left[\left(k^{2}-v^{2}\right)^{\frac{1}{2}} \cos \left\{\left(k^{2}-v^{2}\right)^{\frac{1}{2}}(y+n)\right\}-K \sin \left\{\left(k^{2}-v^{2}\right)^{\frac{1}{2}}(y+n)\right\}\right]}{k^{2}+k^{2}-v^{2}} \times \\
& \times \exp (-k|x-\xi|) d k+2 \pi i \frac{K}{\left(K^{2}-v^{2}\right)^{\frac{1}{2}}} \exp \left\{-K(y+n)+i\left(K^{2}-v^{2}\right)^{\frac{1}{2}}|x-\xi|\right\} .
\end{aligned}
$$

Considering the continuity of $\Phi(x, y)$ across the gap we obtain the IE for $f(y)$ as

$$
\int_{a}^{b} G(0, y ; 0, n) f(y) d y=-\pi \phi_{0}(0, n)
$$

where $f(y) \sim 0\left(y^{2}-a^{2}\right)^{-\frac{1}{2}}$ as $y \rightarrow a$ and $f(y) \sim 0\left(b^{2}-y^{2}\right)^{-\frac{1}{2}}$ as $y \rightarrow b$.

3. SOLUTION OF THE INTEGRAL EQUATION.

To solve the IE (2.11) we first expand its Kernel in terms of $\varepsilon$ where $\varepsilon=\sin \alpha$ so that $0 \leqq \varepsilon<1 . \varepsilon=0$ corresponds to the case of a normally incident wave for which the solution is supposed to be known. This technique is used earlier successfully by Mandel and Goswami [4], [5], [6], [7] in connection with the scattering of an obliquely incident wave train by obstacles of some particular geometrical shapes. Now following Mandal and Goswami [6]

$$
\begin{aligned}
G(0, y ; 0, n) & =G_{o}(0, y ; 0, \eta)+\varepsilon^{2} \ln \varepsilon G_{1}(0, y ; o, n) \\
& +\varepsilon^{2} G_{2}(0, y ; o, n)+0\left(\varepsilon^{4} \ln \varepsilon, \varepsilon^{4}\right)
\end{aligned}
$$


where

$$
\begin{aligned}
G_{0}(0, y ; 0, n) & =2 \int_{0}^{\infty} \frac{(K \sin k y-k \cos k y)(K \sin k n-k \cos k n)}{k\left(k^{2}+K^{2}\right)} d k \\
& +2 \pi i \exp \{-K(y+n)\}, \\
G_{1}(0, y ; 0, n) & =(1-K y)(1-K n), \\
G_{2}(0, y ; 0, n) & =-K^{2} \int^{\infty} \frac{(K \sin k y-k \cos k y)(K \sin k n-k \cos k)}{k^{3}\left(k^{2}+K^{2}\right)} d k \\
& +(1-K y)(1-K n)\{\delta+\sqrt{2}-1-\ln (\sqrt{2}+1)\} \\
& +\pi i \exp \{-K(y+n)\} \\
& =\sum_{n=2}^{\infty}(-1)^{n} \frac{1.3 .5 \ldots(2 n-1)}{2^{n} n !} \frac{1}{2 n-2},
\end{aligned}
$$

etc.

Thus to solve the IE (2.11) we assume a similar expansion for $f(y)$ given by

$$
f(y)=f_{0}(y)+\varepsilon^{2} \ln \varepsilon f_{1}(y)+\varepsilon^{2} f_{2}(y)+0\left(\varepsilon^{4} \ln \varepsilon, \varepsilon^{4}\right)
$$

where $f_{n}(y)$ satisfies the IE

$$
\int_{a}^{b} f_{n}(y) G_{0}(0, y ; 0, n) d y=u_{n}(n), a<n<b, n=0,1,2, \ldots
$$

Here

$$
u_{0}(n)=-\pi \exp (-K n)
$$

and

$$
u_{n}(n)=-\int_{a}^{b} f_{0}(y) G_{n}(0, y ; 0, n) d y, n=1,2, \ldots \text {, }
$$

and $f_{n}(y)$ has integrable singularities at the two ends. Closed form solution of (3.4) is not possible, and instead, as in Packham and Williams [2], we solve it approximately by exploiting the assumption that the depth of submergence $h\left(=\frac{a+b}{2}\right)$ of the midline of the gap is large compared to the breadth $b-a=2 c$ say.

Now the kernel in the IE (3.4) can be written as

$$
G_{0}(0, y ; 0, n)=-\ln |y-n|+g(0, y ; 0, n)
$$

where $g(0, y ; 0, n)$ is regular. Then $(3.4)$ is

$$
\int_{a}^{b} f_{n}(y) \ln |y-n| d y=-u_{n}(n)+\int_{a}^{b} f_{n}(y) g(o, y ; o, n) d y, a<n<b .
$$

Since $2 c$ is small compared to $h$, we can approximate the right side of (3.6) by its value at $n=h$, so that $(3.6)$ reduces to

$$
\int_{a}^{b} f_{n}(y) \ln |y-n| d y=A_{n}, a<n<b,
$$

where $A_{n}=-u_{n}(h)+g(o, h ; 0, h) c_{n}, n=0,1,2, \ldots$ 
and $c_{n}=\int_{a}^{b} f_{n}(y) d y$.

Now the solution of $(3.7)$ when $f_{n}(y)$ has integrable singularities is given by (cf. Cooke [9])

$$
f_{n}(y)=\frac{A_{n}}{\pi\{(y-a)(b-y)\}^{\frac{1}{2}}} \cdot \frac{1}{\ln \left(\frac{b-a}{4}\right)}
$$

provided $\frac{b-a}{4} \neq 1$.

Thus $\quad c_{n}=-\frac{u_{n}(h)}{\ln \frac{b-a}{4}-g(o, h ; o, h)}, n \geqq 0$.

For $n=0, \quad c_{0}=-\frac{u_{0}(h)}{\ln \frac{b-a}{4}-g(0, h ; 0, h)}$.

Also $u_{n}(h)=-\int_{a}^{b} f_{o}(y) G_{n}(o, y ; o, h) d y$

$$
=-G_{n}(0, h ; 0, h) c_{0},
$$

so that $c_{n}=\frac{G_{n}(o, h ; o, h) c_{o}}{\ln \frac{b-a}{4}-g(0, h ; o, h)}, n>0$.

4. TRANSMISSION AND REFLECTION COEFFICIENTS.

Making $\xi \rightarrow+\infty$ in (2.7), $\xi \rightarrow-\infty$ in (2.8) and noting the form of $G(x, y ; \xi, n)$ in (2.9) as $|\xi| \rightarrow \infty$, and using (2.5) and (2.6) we obtain respectively

$$
\begin{gathered}
T=-2 i\left(1-\varepsilon^{2}\right)^{-\frac{1}{2}} \int_{a}^{b} \exp (-K y) f(y) d y, \\
R=1+2 i\left(1-\varepsilon^{2}\right)^{-\frac{1}{2}} \int_{a}^{b} \exp (-K y) f(y) d y,
\end{gathered}
$$

so that $T+R=1$. Noting (3.3) we can expand $T$ and $R$ as

$$
\begin{aligned}
& T=T_{0}+\varepsilon^{2} \ln \varepsilon T_{1}+\varepsilon^{2} T_{2}+0\left(\varepsilon^{4} \ln \varepsilon, \varepsilon^{4}\right) \\
& \mathrm{R}=\mathrm{R}_{\mathrm{o}}+\varepsilon^{2} \ln \varepsilon \mathrm{R}_{1}+\varepsilon^{2} \mathrm{R}_{2}+0\left(\varepsilon^{4} \ln \varepsilon, \beta^{4}\right) \\
& \text { so that } \mathrm{T}_{\mathrm{o}}+\mathrm{R}_{\mathrm{o}}=1, \mathrm{~T}_{1}+\mathrm{R}_{1}=0, \mathrm{~T}_{2}+\mathrm{R}_{2}=0 \text {, etc. } \\
& \text { Now } \quad I_{0} \simeq-21 \exp (-K h) c_{0} \text {, } \\
& \mathrm{T}_{1} \simeq-21 \exp (-\mathrm{Kh}) \mathrm{c}_{1} \text {, } \\
& T_{2} \simeq-2 i \exp (-K h)\left(\frac{c_{o}}{2}+c_{2}\right) \text {, } \\
& \text { etc. }
\end{aligned}
$$


Following Evans [10]

$$
\begin{gathered}
g(0, h ; 0, h) \approx \ln (2 h)-2\left\{\bar{E}_{i}(2 K h)-\pi i\right\} \exp (-2 K h) \\
\text { where } \bar{E}_{i}(x)=f_{-\infty}^{x}\left(e^{t} / t\right) d t \\
\text { Thus } T_{0}=-\frac{i}{-i+\frac{1}{2 \pi} e^{2 K h} \ln \frac{c}{4 h}+\frac{1}{\pi} \bar{E}_{i}(2 K h)}, \\
T_{1} \simeq-\frac{1}{2 \pi i}(1-K h)^{2} \exp (-2 K h) T_{0}^{2}, \\
T_{2} \simeq \frac{T_{0}}{2}\left[1-\frac{T_{0}}{i \pi} \exp (2 K h) G_{2}(0, h ; 0, h)\right]
\end{gathered}
$$

etc.

We may note that $\left|\mathrm{T}_{\mathrm{o}}\right|$ coincide with the result given by Tuck [1] and Packham and Williams [2] for a narrow gap.

5. DISCUSSION.

The approximate expressions for the transmission and reflection co-efficients are obtained here under the assumption that the gap is narrow. For a fixed value of the ratio $\frac{2 c}{h}=0.15$ (as considered by Packham and Williams [2]) numerical calculations are performed for $\mathrm{Kh}=0.1,0.2,0.4,0.6,0.8,1.0,1.2,1.4$ and 1.6 and the angle of incidence $\alpha=0^{\circ}, 5^{\circ}, 10^{\circ}, 15^{\circ}, 20^{\circ}, 25^{\circ}$ and $30^{\circ} .|\mathrm{T}|$ and $|\mathrm{R}|$ are graphically represented against $\alpha$ up to $30^{\circ}$ for the different values of $\mathrm{Kh}$ mentioned above (cf. fig.1,2 and 3). It is observed that for a fixed $\alpha$, the transmission co-efficient first increases while the reflection co-efficient decreases and then the transmission co-efficient decreases while the reflection co-efficient increases for different values of $\mathrm{Kh}$. This has already been demonstrated in the result of Packham and Williams [2] and Tuck [1] for the case of normal incidence and infinite depth of water. However for a fixed $\mathrm{Kh}$, the transmission co-efficient increases and the reflection co-efficient decreases as a increases. This type of phenomenon was also observed in connection with the scattering of an obliquely incident surface wave train by a fixed vertical partially immersed barrier (cf. Mandal and Goswami [4], Evans and Morris [11]), a submerged plate (cf. Mandal and Goswami [5]), a submerged barrier extended infinitely downwards (cf. Manda1 and Goswami [6]) and a half immersed circular cylinder (cf. Mandal and Goswami [7]). A11 the curves for different fixed values of $\mathrm{Kh}$ exhibit the behavior that more energy is transmitted through the gap, when the wave train is obliquely incident than when it is normally incident on a barrier with a narrow gap.

For other small values of $\frac{2 c}{h}$, numerical calculations are also performed and similar qualitative nature of the transmission and reflection co-efficients are observed. It may be noted that we have restricted our analytical as well as numerical calculations correct up to four decimal places and up to $\alpha=30^{\circ}$. However as $0 \leqq \varepsilon=\sin \alpha<1$, in principle, there is no difficulty to make calculations for more values of $\alpha$. However in that case we need to consider higher order terms involving $\varepsilon^{n}$ and $\varepsilon^{n}$ ln $\varepsilon(n \geqq 4)$ in the expansion of the kernel of the IE and similar terms in $\mathrm{f}(\mathrm{y}), \mathrm{T}$ and $\mathrm{R}$. 


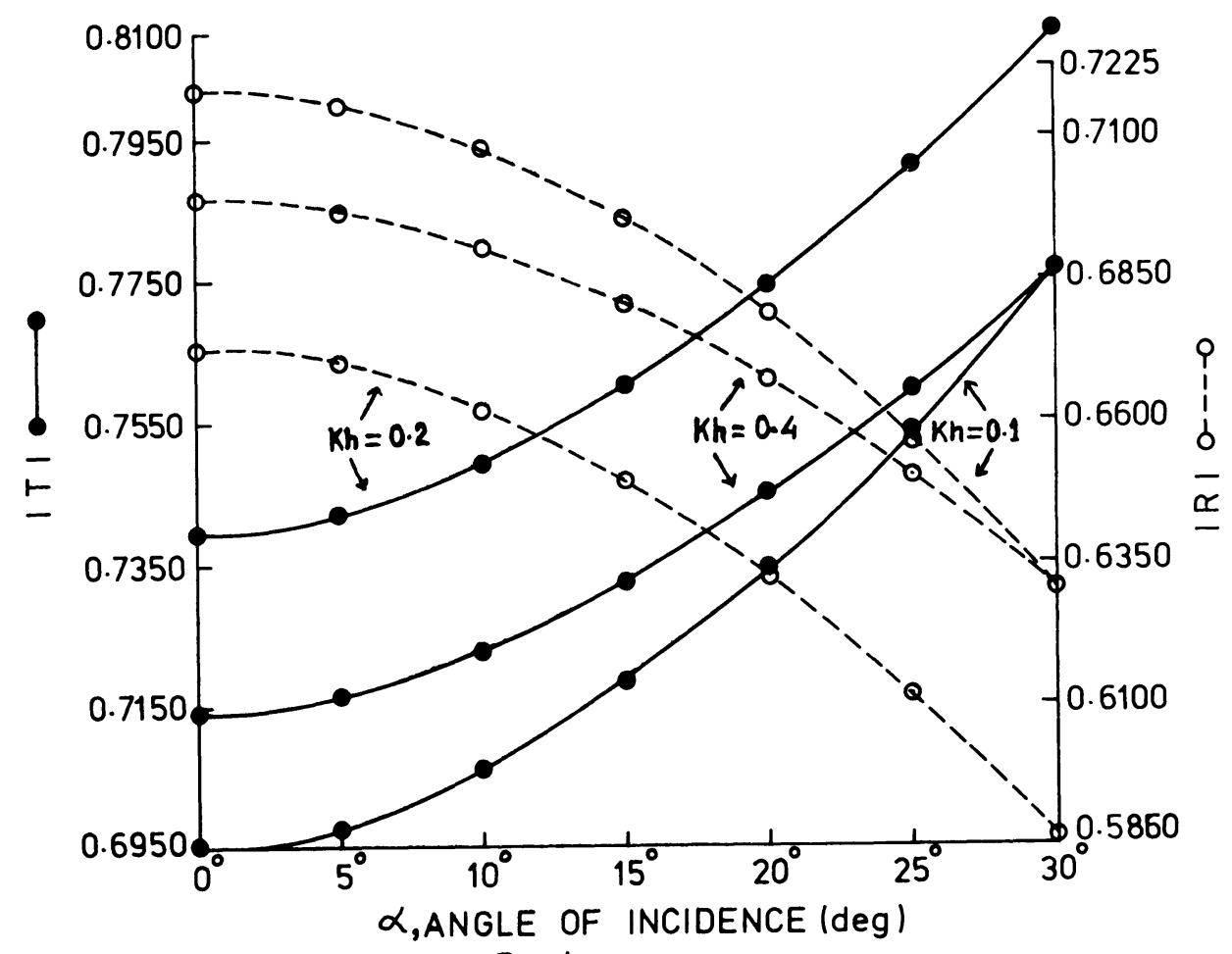

Fig. 1

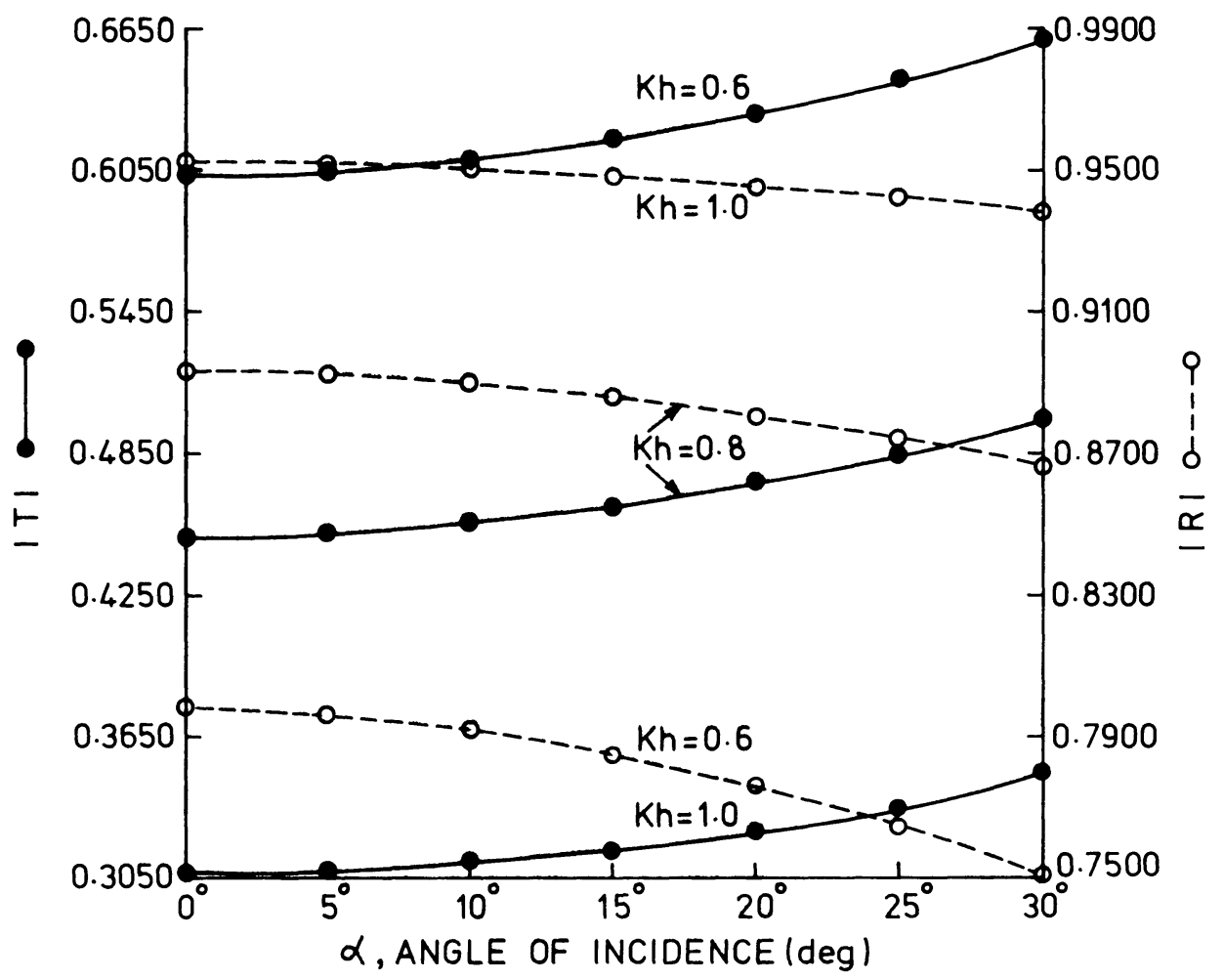

Fig. 2 


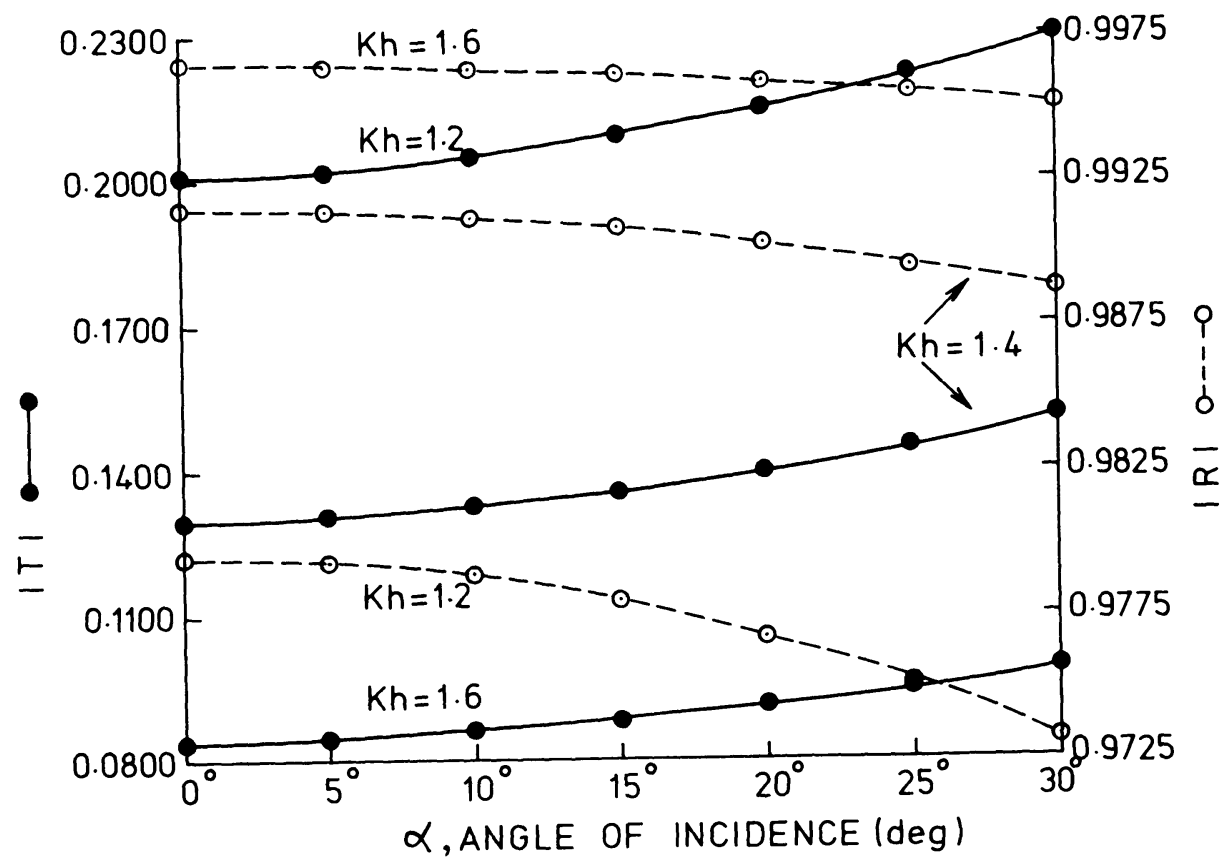

Fig. 3

ACKNOWLEDGEMENT. This work was carried out under the project No.F.8-8/85(SR III) dt. 3 May, 1985, sponsored by U.G.C., New Delhi.

REFERENCES

1. TUCK, E.0. Transmission of water waves through small aperatures, J. Fluid Mech. $49(1971), 65-74$.

2. PACKHAM, B.A. and WILLIAMS, W.E. A note on the transmission of water waves through small aperatures, J. Inst. Maths. Applics. 10 (1972), 176-184.

3. PORTER, D. The transmission of surface waves through a gap in a vertical barrier, Proc. Camb. Phil. Soc. 71 (1972), 411-421.

4. MANDAL, B.N. and GOSWAMI, S.K. A note on the diffraction of an obliquely incident surface wave by a partially immersed fixed vertical barrier, App1. Sc1. Res. 40 (1983), 345-353.

5. MANDAL, B.N. and GOSWAMI, S.K. The scattering of an obliquely incident surface wave by a fixed vertical plate, J. Math. Phys. 25 (1984), 1780-1783.

6. MANDAL, B.N. and GOSWAMI, S.K. A note on the scattering of surface waves obliquely incident on a submerged fixed vertical barrier, J. Phys. Soc. Japan 53 (1984), 2980-2987.

7. MANDAL, B.N. and GOSWAMI, S.K. Scattering of surface waves obliquely incident on a fixed half-immersed circular cylinder, Math. Proc. Camb. Phil. Soc. 96 (1984), 359-369.

8. LEVINE, H. Scattering of surface waves by a submerged circular cylinder, J. Math. Phys. 6 (1965), 1231-1243.

9. COOKE, J.C. The solution of some integral equations and their connection with dual integral equations and series, Glasgow Math. J. 11 (1970), 9-20.

10. EVANS, D.V. Water-wave transmission through barriers with small gaps, J. Engg. Math. 11 (1977), 1-10.

11. EVANS, D.V. and MORRIS, C.A.N. The effect of a fixed vertical barrier on obliquely incident surface waves in deep water, J. Inst. Maths. Applies. g. (1972), 198-204. 


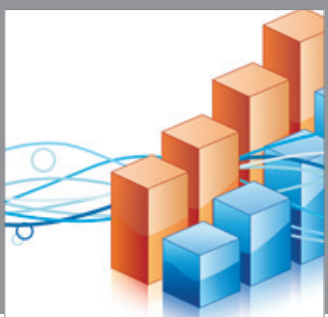

Advances in

Operations Research

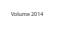

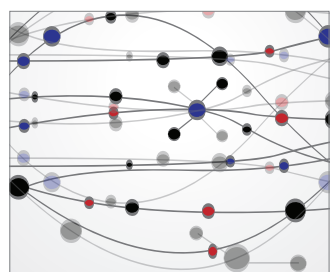

\section{The Scientific} World Journal
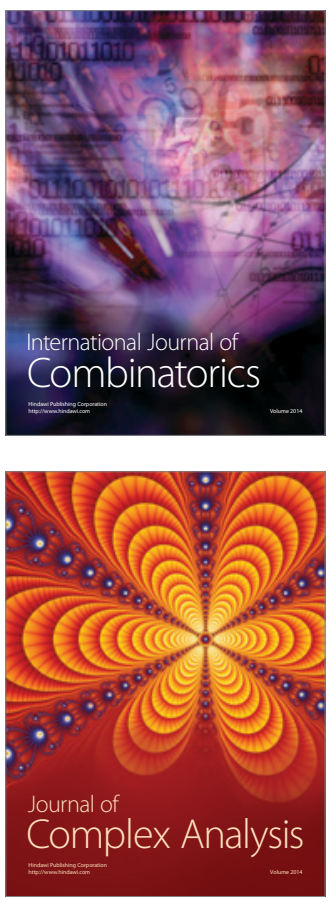

International Journal of

Mathematics and

Mathematical

Sciences
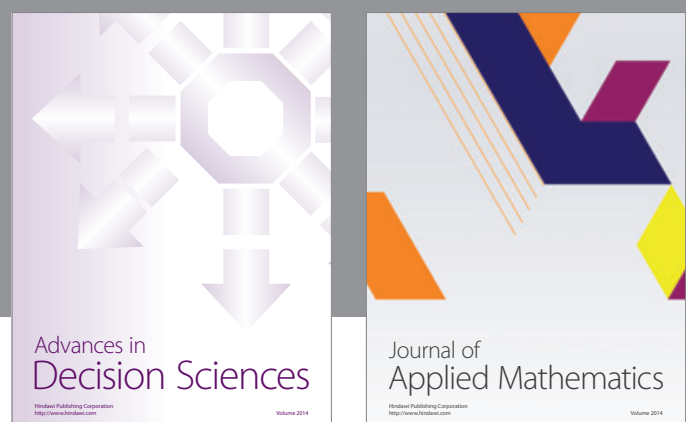

Journal of

Applied Mathematics
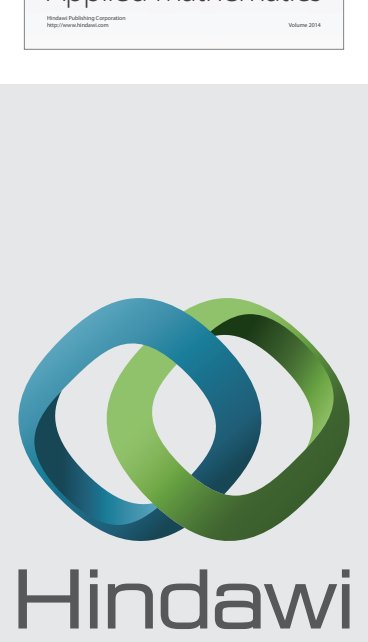

Submit your manuscripts at http://www.hindawi.com
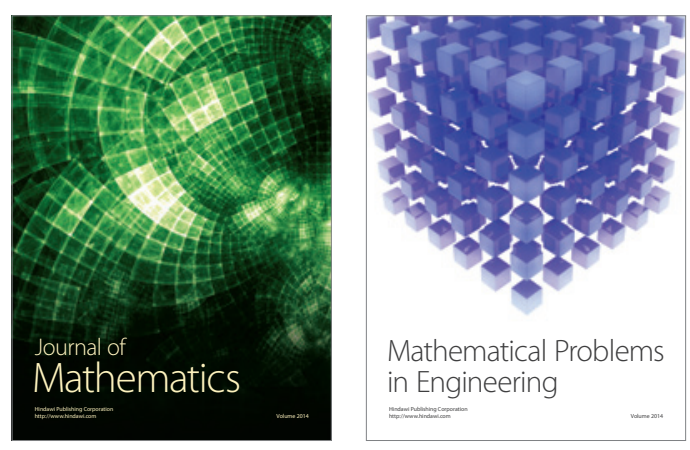

Mathematical Problems in Engineering
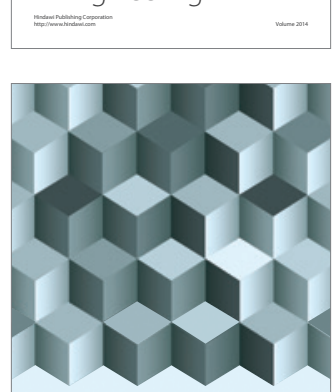

Journal of

Function Spaces
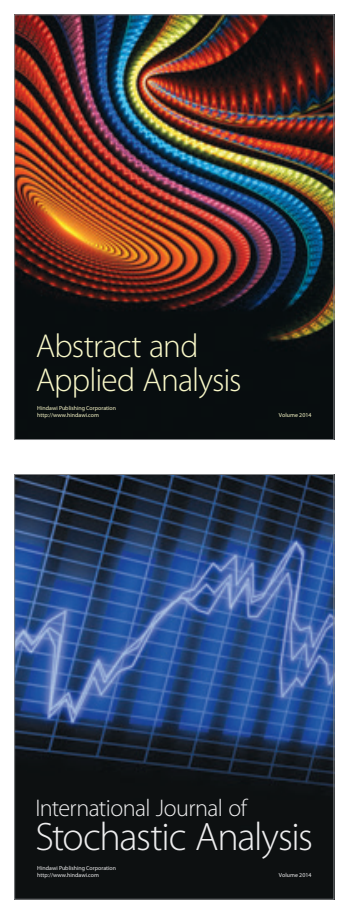

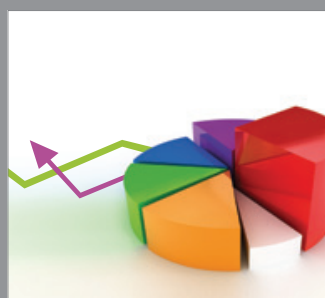

ournal of

Probability and Statistics

Promensencen
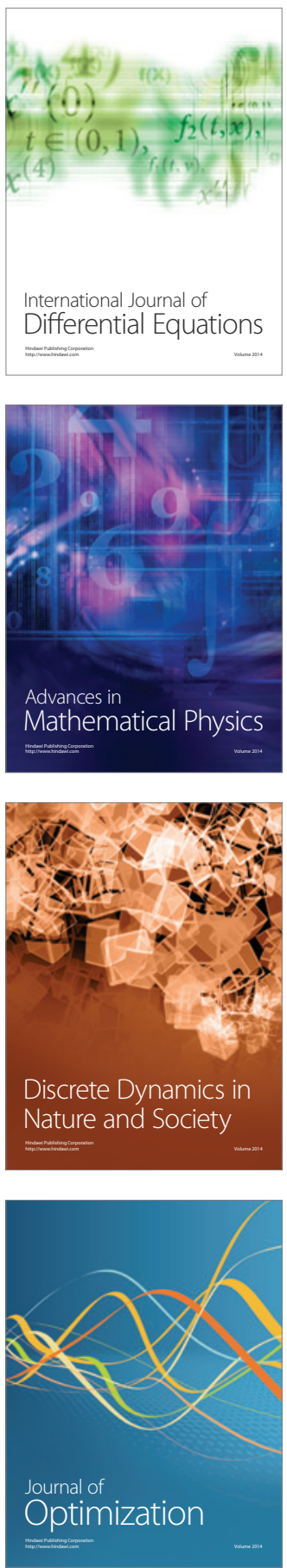